\title{
FERRAMENTA DE APOIO À FORMULAÇÃO SIMBÓLICA DE MODELOS CINÉTICOS MICROBIANOS NÃO-ESTRUTURADOS
}

\author{
Martins, F. P. R. ${ }^{1}$, Barral, M. F. ${ }^{2}$ \\ ${ }^{1}$ IPT - Instituto de Pesquisas Tecnológicas do Estado de São Paulo - Agrupamento de Sistemas \\ de Controle. Email: fmartins@ipt.br \\ ${ }^{2}$ IPT - Instituto de Pesquisas Tecnológicos do Estado de São Paulo - Agrupamento de \\ Biotecnologia. Email: mfbarral@ipt.br
}

\section{Resumo:}

Com o intuito de aprimorar e facilitar as atividades de modelagem, desenvolveu-se um software de apoio à formulação de modelos cinéticos não-estruturados, o qual gera, automaticamente, estruturas matemáticas simbólicas. Estas são montadas a partir de seleções que o usuário realiza utilizando as informações que detém sobre os principais fenômenos que afetam a cinética dos processos fermentativos. Para, tanto, são combinadas variadas fontes de conhecimento, representadas através de cláusulas Prolog, e que abrangem os seguintes aspectos do processo: tipos de sistema de cultivo, reações estequiométricas, fenômenos de limitação, inibição, morte celular, manutenção microbiana, decomposição de substratos e formação de produtos. O resultado final são conjuntos de equações diferenciais simbólicas, com parâmetros conhecidos e/ou a estimar, as quais podem ser exportadas e utilizadas em programas de determinação e ajuste de parâmetros cinéticos e de simulação, otimização e projeto de reatores bioquímicos.

Palavras-Chave: processos fermentativos, sistemas especialistas, lógica.

\begin{abstract}
:
A program has been developed to help and improve the mathematical modelling of microbiological systems using kinetic non_structured models. This computational tool generates symbolic mathematical structures based on the user choices concerning the main fermentation process kinetic phenomena. Using Prolog clauses to represent the expert biochemical knowledge, symbolic mathematical modelling encompasses the following aspects of the process: substrates, products and microorganisms involved, types of cultivation systems, stoichiometry of the overall reactions, substrate limitation and inhibition, product inhibition, microbial death and maintenance, substrate decomposition and product formation kinetics. The program generates sets of symbolic differential equations which can be exported and used in programs to fit kinetic parameters for simulation, optimisation and design of biochemical reactors.

Keywords: fermentation processes, expert systems, logic
\end{abstract}

Endereço para contato:

${ }^{1}$ IPT - Instituto de Pesquisas Tecnológicas do Estado de São Paulo - Agrupamento de Sistemas de Controle. Cidade Universitária, Butantã, São Paulo, tel: (011) 3767-4510

${ }^{2}$ IPT - Instituto de Pesquisas Tecnológicos do Estado de São Paulo - Agrupamento de Biotecnologia. Cidade Universitária, Butantã, São Paulo, tel: (011) 3767-4543 


\section{Introdução}

Ferramentas computacionais de apoio, capazes de disponibilizar vários métodos para análise e projeto de processos, sem, todavia, prescindir da interação com o usuário final, constituem-se em um modelo básico para a arquitetura dos modernos programas voltados à solução de problemas de engenharia. Essa tendência tem sido reforçada pelo surgimento de novos recursos computacionais bem como pela constatação de que a sistematização do conhecimento é fator indispensável para o desenvolvimento industrial.

Em paralelo, a modelagem automática do raciocínio de um especialista em uma dada atividade de engenharia, é uma das áreas onde as técnicas de inteligência artificial têm se mostrado mais bem sucedidas (Nilsson, 1998), sendo a engenharia de processos, em particular, um pródigo campo de provas para o projeto de sistemas supervisores inteligentes baseados na representação e integração de fontes de conhecimento (Sthephanopoulos e Han, 1996).

A construção de modelos matemáticos é atividade essencial para o desenvolvimento de processos fermentativos. Entretanto

\section{Metodologia}

Considerando-se que o conhecimento necessário para a construção dos modelos simbólicos cinéticos não se acha descrito explicitamente na literatura, na forma de um algoritmo, mas que, ao contrário, cada novo problema requer a "reunião de especialistas em torno de uma mesa redonda", onde cada qual oferece contribuição específica e incremental à resolução do problema, iniciou-se o projeto de desenvolvimento do sistema especialista da forma clássica - ou seja, através de atividades de engenharia de conhecimento (Hayes-Roth, 1983).

Assim, após a realização de uma série de entrevistas com os diversos pesquisadores do Agrupamento de Biotecnologia do IPT envolvidos no projeto, foi possível: 1) fixar a gama de problemas a serem abordados pelo sistema; 2) determinar o espectro geral de soluções elementares para a resolução desses problemas; 3 ) existem poucos profissionais especialistas na análise e modelagem da cinética de processos, sendo que a maior parte dos que atuam na área encontra dificuldade na manipulação das informações matemáticas, razão pela qual o processo de geração de modelos é lento e sujeito a erros.

Com o propósito de minimizar tais dificuldades, desenvolveu-se uma ferramenta computacional que encapsula o conhecimento de um engenheiro de processos fermentativos com habilidade em álgebra e em construção de equações diferenciais de primeira ordem. Dotado desse recurso, a tarefa do usuário torna-se muito mais simples: cabe-lhe apenas fornecer as características relevantes do processo, que, organizadas pelo sistema especialista, produzem, na forma simbólica, as fórmulas e equações diferenciais descritivas do processo em foco.

A ferramenta implementada permite rapidamente formular modelos cinéticos microbianos passíveis de serem ajustados e testados pelo usuário e, ao mesmo tempo, promove a sistematização do conhecimento especializado e sua posterior utilização por outros grupos de pesquisadores.

compreender e então descrever, esquematicamente, o raciocínio utilizado pelos vários especialistas na resolução dos sub-problemas de sua especialidade.

Conclusão básica dessa etapa inicial do projeto foi o estabelecimento de que $o$ sistema especialista deveria sistematizar a experiência dos pesquisadores na modelagem, otimização e controle de processos fermentativos descritos por modelos não-estruturados disponíveis na literatura especializada. Apesar das evidentes limitações que são inerentes a esses modelos (Nielssen e Villandsen, 1994), os mesmos foram adotados por causa de sua importância clássica e, também, por possuírem estruturas algébricas que favorecem a posterior análise dos resultados.

A etapa subseqüente do ciclo de desenvolvimento do sistema iniciou-se com a seleção do paradigma de representação 
de conhecimento - no caso, lógica de primeira ordem, implementada através de cláusulas Prolog. Esta escolha foi motivada, em grande parte, pelas facilidades que essa linguagem oferece para a construção de interpretadores lingüísticos e, também, porque favorece a modelagem de variados tipos de raciocínio (Bratko, 1990). Em particular, adotou-se o dialeto Visual Prolog (PDC, 2002), pois este disponibiliza ao usuário os recursos gráficos do ambiente Windows.

Tomadas essas decisões procedeu-se à programação propriamente dita, gerando-se um extenso código composto de cláusulas representativas de conhecimento especializado em geração de modelos, cláusulas de controle, cláusulas de interação gráfica e cláusulas auxiliares diversas.

Dentre as hipóteses admitidas para a modelagem dos processos, merecem destaque:

- uso exclusivo de reator de mistura perfeita;

- utilização de um único microorganismo;

Quanto à composição do processo, admitiu-se que este deva ser o resultado de combinações dos seguintes fenômenos:

- consumo de substratos para crescimento;

- morte celular;

- consumo de substratos para manutenção;

- decomposição de substratos;

- inibição por produto;

- formação de produto.

Além disso, incluiu-se na modelagem os conceitos de substratos melhoradores, essenciais e preferenciais (Bonomi e Schimidell, 2001).

A interface com o usuário foi projetada com as opções necessárias para que se possa caracterizar livremente o processo a ser modelado pelo sistema. Assim, estabeleceu-se para o usuário as seguintes "missões":

- Cadastrar microorganismos e substâncias;

- Escolher o sistema de cultivo - batelada, batelada alimentada ou regime contínuo;

- Selecionar microorganismo, substratos e produtos;

- Fornecer os valores iniciais das variáveis de estado automaticamente geradas pelo sistema;

- Fornecer as vazões de entrada/saída;

- Definir as equações estequiométricas para consumo de substratos e formação de produtos;

- Definir o papel - melhorador ou essencial desempenhado por cada substrato;

- Definir as prioridades para o consumo dos diversos substratos;

- Fornecer valores (ou definir que se trata de constantes a determinar) para os vários parâmetros dos modelos não-estruturados representativos do fenômeno em foco;

Enquanto que, para o sistema especialista, foram definidas as seguintes tarefas básicas:

- Criar automática e consistentemente as variáveis de estado;

- Criar, em função do sistema de cultivo, um modelo consistente para as correntes de alimentação e refugo do fermentador;

- Criar grupos $G_{i}$ de substratos $S_{a}, S_{b}, \ldots, S_{n}$ simultaneamente consumidos, bem como uma escala de prioridades ordenando os vários $G_{i}$ 's segundo a ordem de consumo preferencial;

- Prover modelos matemáticos adequados, na forma simbólica para cada fenômeno considerado, a saber: consumo de um substrato $S_{i}$ para crescimento, consumo de um substrato $S_{i}$ para manutenção, decomposição de um substrato $S_{i}$, morte celular, inibição do crescimento por um produto $P_{j}$, consumo de um substrato $S_{i}$ para formação de um produto $P_{i}$. 
- Gerar, para cada grupo $G_{i}$ de substratos, as correspondentes expressões simbólicas das velocidades específica de crescimento $\left(\mu_{X}^{j}\right)$ e de morte celular $\left(\mu_{X m}{ }^{j}\right)$, se tal fenômeno ocorrer;

- Associar a cada uma das expressões de $\mu_{x}^{i}$ anteriores, as expressões simbólicas dos fatores de inibição por produto (se esse fenômeno ocorrer) relativas aos produtos gerados durante a mesma fase de consumo do grupo $G_{i}$ considerado;

- Gerar, para cada produto $P_{i}$, a expressão da velocidade específica de formação $\mu_{P}{ }^{i}$, na forma simbólica;

- Gerar, para cada grupo de substratos $G_{i}$, o correspondente sistema de equações diferenciais que descrevem a variação temporal das variáveis de estado;

- Produzir arquivos de saída contendo, para cada grupo $G_{i r}$ as expressões simbólicas dos parâmetros cinéticos e das equações diferenciais.

\section{Resultados}

Para a validação do software, foram realizados testes abrangentes envolvendo processos com múltiplas combinações de fenômenos. Os modelos simbólicos gerados pelo sistema foram, então, comparados com modelos produzidos manualmente. Com isso, detectaram-se algumas inconsistências geradas por falhas na modelagem do raciocínio. Após a correção dessas falhas, novas baterias de testes foram sucessivamente aplicadas ao sistema, e, como era de se esperar, à medida que os modelos se tornavam mais e mais complexos, surgiam novas inconsistências, mas, agora, motivadas por falha na elaboração manual dos modelos simbólicos.

A título de ilustração, apresentam-se abaixo alguns exemplos de processos que foram modelados pelo sistema:

Caso 1: Cultivo em batelada com produção de biomassa limitada por dois substratos essenciais $\left(S_{1}\right.$ e $\left.S_{2}\right)$, ocorrendo consumo de ambos para manutenção celular e crescimento inibido pelo substrato $S_{1}$.

Para esse caso, geraram-se as equações:

1. eqdif("G1(S1,S2)", "dS1dt","-1/YXvS1*dXvdt- msS1*Xv"

1. eqdif("G1(S1,S2)", "dS2dt","-1/YXvS2*dXvdt- msS2*Xv")

1. eqdif("G1(S1,S2)","dXvdt","muX*Xv")

1. $\operatorname{mux}(" \mathrm{G} 1(\mathrm{~S} 1, \mathrm{~S} 2) "$,

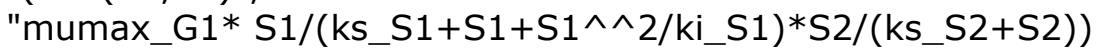

que correspondem ao seguinte sistema de equações diferenciais:

$$
\begin{aligned}
& \frac{d S_{1}}{d t}=-\frac{1}{Y_{X_{v} / S_{1}}} \cdot \frac{d X_{v}}{d t}-m s_{1} \cdot X_{v} \\
& \frac{d S_{2}}{d t}=-\frac{1}{Y_{X_{v} / S_{2}}} \cdot \frac{d X_{v}}{d t}-m s_{2} \cdot X_{v} \\
& \frac{d X_{v}}{d t}=\mu_{\max }^{G_{1}} \cdot \frac{S_{1}}{k s_{S_{1}}+S_{1}+S_{1}^{2} / k i_{S_{1}}} \cdot \frac{S_{2}}{k s_{S_{2}}+S_{2}} \cdot X_{v} \text { onde: }
\end{aligned}
$$

- $\mathrm{G}_{1}\left(\mathrm{~S}_{1}, \mathrm{~S}_{2}\right)$ refere-se ao grupo de dois substratos $\mathrm{S}_{1}$ e $\mathrm{S}_{2}$ que são consumidos para a produção de biomassa $X$.

- $Y_{X_{v} / S_{i}}$ é o coeficiente de rendimento da reação de produção de biomassa a partir do substrato $S_{i}$. 
- $m s_{S_{i}}$ é o coeficiente de manutenção celular no substrato $S_{i}$.

- $\quad \mu_{\max }^{G_{i}}, k s_{S_{i}}$ e $k i_{S_{i}}$ são coeficientes utilizados nos modelos cinéticos de Andrews (1968) e Monod (1942) e que, no caso, foram selecionados pelo usuário para representar, respectivamente, os processos de formação de biomassa limitada e inibida por $\mathrm{S}_{1} \mathrm{e}$ limitada por $\mathrm{S}_{2}$.

- $X_{v}, S_{1}$ e $S_{2}$ são as variáveis de estado do sistema representando, respectivamente, concentrações de células, substrato $S_{1}$ e substrato $S_{2}$.

Caso 2: Cultivo em batelada com crescimento celular limitado pelos substratos $S_{1}$ e $S_{2}$, formação de produto não-associada (Luedeking e Piret, 1959) ao crescimento utilizando um substrato $S_{3}$. Na primeira fase do processo o crescimento é limitado e inibido pelo substrato $S_{1}$ (essencial) segundo o modelo de Andrews, $S_{2}$ é um substrato melhorador (Dunn et al, 1992).) representado pelo modelo de Monod e sujeito a decomposição. A segunda fase refere-se à formação de produto não-associada.

Para esse caso, geraram-se as equações:

1. eqdif("G1(S1,S2)","dS1dt","- 1/YXvS1*dXvdt")

1. eqdif("G1(S1,S2)","dS2dt","-1/YXvS2*dXvdt - KdS2*S2")

1. eqdif("G1(S1,S2)","dXvdt","muX*Xv")

1. eqdif("G2(S3)","dP1dt","muP1*Xv")

1. eqdif("G2(S3)","dS3dt","-1/YP1S3*dP1dt")

1. eqdif("G2(S3)","dXvdt","0")

1. $\operatorname{mux}\left(" \mathrm{G} 1(\mathrm{~S} 1, \mathrm{~S} 2) ", "\left(\operatorname{mumax} \_\mathrm{G} 1+\mathrm{mu} \_\mathrm{S} 2 * \mathrm{~S} 2 /\left(\mathrm{ks} \_\mathrm{S} 2+\mathrm{S} 2\right)\right) * \mathrm{mu} \_\mathrm{S} 1 * \mathrm{~S} 1 /\left(\mathrm{ks} \_\mathrm{S} 1+\mathrm{S} 1+\mathrm{S} 1 \wedge \wedge 2\right.\right.$ /ki_S1)")

1. mup("P1","BetaP1")

que correspondem a dois sistemas distintos de equações diferenciais, o primeiro dos quais regendo o fenômeno de formação de biomassa a partir dos substratos $S_{1}$ e $S_{2}$, ou seja:

$$
\begin{aligned}
& \frac{d S_{1}}{d t}=-\frac{1}{Y_{X_{v} / S_{1}}} \cdot \frac{d X_{v}}{d t} \\
& \frac{d S_{2}}{d t}=-\frac{1}{Y_{X_{v} / S_{2}}} \cdot \frac{d X_{v}}{d t}-k d_{2} \cdot S_{2} \\
& \frac{d X_{v}}{d t}=\left[\mu_{\max }^{G_{1}}+\mu_{S_{2}} \cdot \frac{S_{2}^{2}}{k s_{S_{2}}+S_{2}^{2}} \cdot \mu_{S_{1}} \cdot \frac{S_{1}^{2}}{k s_{S_{1}}+S_{1}}\right] X_{v}
\end{aligned}
$$

e o segundo, o processo de formação de produto não associado ao crescimento de biomassa, a partir do substrato $\mathrm{S}_{3}$.

$$
\begin{aligned}
& \frac{d P_{1}}{d t}=\beta_{P_{1}} \cdot X_{v} \\
& \frac{d S_{3}}{d t}=-\frac{1}{Y_{P_{1} / S_{3}}} \cdot \frac{d P_{1}}{d t} \\
& \frac{d X_{v}}{d t}=0
\end{aligned}
$$

onde:

- $\mathrm{G}_{1}\left(\mathrm{~S}_{1}, \mathrm{~S}_{2}\right)$ refere-se ao grupo de dois substratos $S_{1}$ e $S_{2}$ que são consumidos preferencialmente pelo microorganismo para a produção de biomassa $X_{v}$.

- $k d_{2}$ é o coeficiente de decomposição do substrato $S_{2}$. 
- $\quad \mu_{\max }^{G_{i}}, \mu_{S_{i}}$ e $k s_{S_{i}}$ são coeficientes utilizados no modelo cinético resultante da combinação dos modelos de Andrews e Monod que, no caso, foram selecionados pelo usuário para representar, respectivamente, os processos de formação de biomassa limitada e inibida por $\mathrm{S}_{2}$ e limitada por $\mathrm{S}_{1}$.

- $\quad \beta_{P 1}$ é o coeficiente de formação do produto $P_{1}$ não associado ao crescimento de biomassa.

- $Y_{P_{1} / S_{3}}$ é o coeficiente de rendimento da reação de produção de $\mathrm{P}_{1}$ a partir do substrato $\mathrm{S}_{3}$.

Caso 3: Batelada alimentada com crescimento celular utilizando dois substratos $S_{1}$ e $S_{2}$, sendo que $S_{1}$ é fornecido na forma de uma corrente de alimentação (vide figura 1, abaixo).

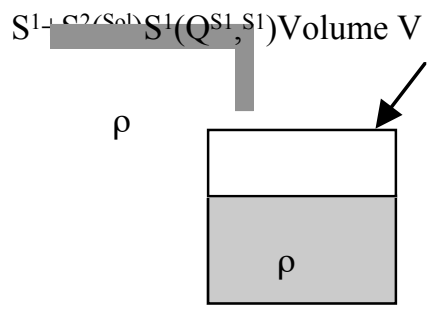

Figura 1: Batelada alimentada com substrato $S_{1}$.

Para esse caso, foram geradas as seguintes equações:

1. eqdif("G1(S1,S2)","dS1dt","-1/YXvS1*dXvdt+QS1e*S1e/V((QS1e*RhoS1e)/Rhosol)/V*S1")

1. eqdif("G1(S1,S2)","dS2dt","-1/YXvS2*dXvdt - ((QS1e*RhoS1e)/Rhosol)/V*S2")

1. eqdif("G1(S1,S2)","dVdt","(QS1e*RhoS1e)/Rhosol")

1. eqdif("G1(S1,S2)","dXvdt","muX*Xv - ((QS1e*RhoS1e)/Rhosol)/V*Xv")

1. $\operatorname{mux}\left(" \mathrm{G} 1(\mathrm{~S} 1, \mathrm{~S} 2) ", " \operatorname{mumax} \_\mathrm{G} 1 * \mathrm{~S} 1 /\left(\mathrm{ks} \_\mathrm{S} 1+\mathrm{S} 1+\mathrm{S} 1 \wedge \wedge 2 / \mathrm{ki} S 1\right) * \mathrm{~S} 2 /\left(\mathrm{ks} \_\mathrm{S} 2+\mathrm{S} 2\right) "\right)$ que correspondem, naturalmente, ao sistema de equações:

$$
\begin{aligned}
\frac{d S_{1}}{d t}= & -\frac{1}{Y_{X_{v} / S_{1}}} \cdot \frac{d X_{v}}{d t}+\frac{Q_{S_{i}}^{e} \cdot S_{1}^{e}}{V}-\frac{Q_{S_{1}}^{e} \cdot \rho_{S_{1}}^{e}}{\rho_{\text {sol }} \cdot V} \cdot S_{1} \\
\frac{d S_{2}}{d t} & =-\frac{1}{Y_{X_{v} / S_{2}}} \cdot \frac{d X_{v}}{d t}-\frac{Q_{S_{1}}^{e} \cdot \rho_{S_{1}}^{e}}{\rho_{s o l} \cdot V} \cdot S_{2} \\
\frac{d V}{d t}= & \frac{Q_{S_{1}}^{e} \cdot \rho_{S_{1}}^{e}}{\rho_{s o l}} \\
\frac{d X_{v}}{d t}= & \mu_{\max }^{G_{1}} \cdot \frac{S_{1}}{k s_{S_{1}}+S_{1}+S_{1}^{2} / k i_{S_{1}}} \cdot \frac{S_{2}}{k S_{S_{2}}+S_{2}} \cdot X_{v} \\
& -\frac{Q_{S_{1}}^{e} \cdot \rho_{S_{1}}^{e}}{\rho_{s o l} \cdot V} \cdot X_{v}
\end{aligned}
$$

onde:

- $Q_{S_{1}}^{e}$ refere-se à vazão de alimentação de $\mathrm{S}_{1}$.

- $\quad \rho_{S_{1}}^{e}$ refere-se à densidade da corrente de alimentação $S_{1}$.

- $\rho_{S o l}$ refere-se à densidade da mistura.

Note que, em todos os casos apresentados anteriormente, as estruturas descritivas das equações diferenciais e das velocidades específicas $\left(\mu_{X}, \mu_{P}\right)$, dependem de parâmetros de modelos clássicos não-estruturados que deverão ser objeto de posterior estimação. 


\section{Conclusões}

O software desenvolvido pode ser um excelente instrumento de apoio à formulação de modelos cinéticos, gerando resultados consistentes e, assim, melhorando o desempenho de profissionais da área quando instados a modelar processos complexos onde a presença de um grande número de variáveis de estado e de combinações de fenômenos diversos dificultam o raciocínio do especialista.

\section{Referências Bibliográficas}

[1].Andrews, J. F. "A mathematical model for the continuous culture of microorganisms utilizing inhibitory substrate", Biotechnology and Bioengineering, 10, pp.707-723, 1968.

[2]. Bonomi, A., Schimidell, W. "Modelagem Matemática e Simulação de Processos Fermentativos",in Biotecnologia Industrial, Schimidell et alli (organizadores), Vol. II, Cap 7, São Paulo, Editora Edgar Blücher Ltda, pp 123-178, 2001.

[3]. Bratko, I. Prolog: Programming for Artificial Intelligence. Massachussets, Addison -Wesley Publ., 1990.

[4]. Dunn, I.J. Heinzle, E., Ingham, J. Prenosil, J.E. Biological reactions Engineering. VCH Verlag $\mathrm{GmbH} .1992$.

[5]. Hayes-Roth, F. Building expert systems. New York, Addison-Wesley Pub., 1983.

[6]. Luedeking, R., Piret, E. L. "A kinetic study of the lactic acid fermentation batch process at controlled pH", Journal of Biochemical and Microbial Technology Engineering, 1, p 393-412. 1959.

[7]. Monod., J. Recherches sur la croissance des cultures bacterienes. Paris: Hermann e Cie. 1942.

[8]. Nielsen, J., Nikolajsen, K. Review and discussion of literature on mathematical models on microbial systems. Copenhagen, Deparment of Bioetecnology, Technical University of Denmark, 1988.

[9]. Nilsson, N. J. Artificial intelligence: a new synthesis. San Francisco, Morgan Kaufmann, Pub., 1998.

[10]. Nielsen, J., Villandsen, J. Bio reactions engineering principles. New York, Plenum Press, 1994.

[11]. PDC, Visual Prolog. Prolog Development Center, www.pdc.dk/vip, 2002.

[12]. Sthephanopoulos, G., Han, C. "Intelligent systems in process engineering: a review", Computers in Chemical Engineering, vol 20, no 6/7, pp 743-91,1996. 